\title{
Equifinality in the modelling of ammonia volatilisation from a flooded rice system
}

\begin{abstract}
Equifinality is the capability of models to produce similar model-output responses. The objective of this study is to use a numerical experimentation to investigate the equifinality of six modules for estimating ammonia (NH3) volatilisation from the floodwater in a rice system. Except for the Chowdary's, all modules can simulate the fluctuating trends of NH3 volatilisation rates. NFLOOD v.1 shows low equifinality to Jayaweera and Mikkelsen's and the regression equations in CERESRice, DSSAT-CSM and APSIM-Oryza in estimating the NH3 volatilisation. The equation in APSIM-Oryza has high equifinality to that in CERES-Rice. Both regression equations have low equifinality to the regression equation in DSSAT-CSM. All three regression equations show low equifinality to Jayaweera and Mikkelsen's. Findings in this study are valuable to make an informed model selection, to interpret model-output responses critically and to improve an existing model by adopting an alternative module without using experimental data explicitly.
\end{abstract}

Keyword: Equifinality; Sensitivity analysis; Model evaluation; Ammonia volatilisation; Flooded rice 